\title{
ESTUDIO DEL MODELO DE PROPAGACIÓN SUZUKI PARA REDES MÓVILES
}

\section{STUDY OF SUZUKI PROPAGATION MODEL FOR MO- BILE NETWORKS}

\section{RESUMEN}

Este artículo presenta brevemente la teoría fundamental del Modelo Suzuki comúnmente usado para modelar la propagación en canales inalámbricos. El modelo toma en cuenta los efectos del sombreado y del multi-trayecto de manera simultánea. Además, se valida el modelo mediante una comparación estadística entre mediciones realizadas en el entorno real y simulaciones realizadas en MATLAB $囚$ a través de la generación de series de tiempo Suzuki distribuidas. Las mediciones se realizaron durante diez horas sobre la banda de frecuencias entre $850 \mathrm{MHz}$ y $900 \mathrm{MHz}$. Con los datos obtenidos en medición durante los primeros cinco minutos, se caracterizó el canal inalámbrico para cada una de las frecuencias muestreadas calculando la media y desviación estándar de la señal recibida. Estos dos parámetros fueron introducidos como variables de entrada para la generación de las respectivas series de tiempo. Para cada frecuencia se realizaron simulaciones prediciendo los niveles de la señal que se obtendrían durante diez horas. Finalmente, se calculó el error cuadrático medio entre los valores medidos y los valores generados en simulación. Los resultados obtenidos demuestran que el modelo es válido como una herramienta de predicción de las características de propagación en comunicaciones móviles.

Palabras clave: comunicaciones móviles, distribución log-normal, distribución Rayleigh, efecto de sombreado, fenómeno de multitrayectoria, modelo suzuki, propagación.

\section{ABSTRACT}

This article briefly presents the fundamental theory of Suzuki model commonly used to model the propagation in wireless channels. The model takes into account the effect of shading and multi-path simultaneously. In addition, the model is validated by a statistical comparison between measurements made in the real environment and simulated in MATLAB $®$ through the generation of Suzuki distributed time series. The measurements were made for ten hours over the frequency band between $850 \mathrm{MHz}$ and $900 \mathrm{MHz}$. With the measurement data obtained during the first five minutes, the wireless channel is characterized for each of the frequencies sampled by calculating the mean and standard deviation of the received sig-

\section{Gina Sierra}

Magister en Ciencias de la Información y las Comunicaciones Docente Politécnico Grancolombiano gsierrap@poligran.edu.co Bogotá, Colombia

Tipo: Artículo de investigación

Fecha de Recepción: Octubre 19 de 2013 Fecha de Aceptación: Diciembre 22 de 2013 
nal. These two parameters were entered as input variables for the generation of the respective time series. For each frequency simulations were performed predicting signal levels that would be obtained for ten hours. Finally, we calculated the mean square error between the measured values and the values generated by simulation. The results obtained show that the model is valid as a tool for prediction of propagation features in mobile communications.

Key words: distribution Rayleigh, lognormal distribution, phenomenon of multipath, propagation, mobile communications, shadowing effect, suzuki model.

\section{INTRODUCCIÓN}

Las comunicaciones móviles han tenido un crecimiento exponencial en los últimos años. Por lo tanto, encontrar el diseño óptimo de una red móvil se ha vuelto relevante. Así por ejemplo, determinar la localización óptima de una estación base, obtener una tasa de datos deseable y estimar la cobertura de la red, son algunos de los aspectos cruciales en el diseño. Además, es deseable evitar realizar una serie de medidas de propagación, las cuales son costosas y consumen mucho tiempo. De esta manera, se han desarrollado diversos modelos de propagación para comunicaciones móviles con el fin de proveer una guía de diseño para sistemas móviles [1]-[3].

Dichos modelos intentan describir los efectos del sombreado y el fenómeno del multi-trayecto por separado o de manera simultánea, tal como lo hace el Modelo Suzuki.

Este artículo está organizado de la siguiente manera: la sección dos presenta el Modelo Suzuki desde el punto de vista estocástico. En la sección tres se presentan algunos trabajos encontrados en la literatura sobre el modelo $\mathrm{Su}$ zuki. En la sección cuatro se muestran las mediciones obtenidas sobre un canal inalámbrico y se describe la forma en que se usa el modelo para predecir y simular las mediciones de propagación del canal. La sección cinco presenta los resultados haciendo una comparación estadística entre los datos medidos en el entorno real y los datos obtenidos con el modelo. Por último, se exponen las conclusiones en la sección seis.

\section{MODELO SUZUKI}

El modelo Suzuki es uno de los modelos más comunes en los sistemas clásicos de macro celdas móviles. Fundamentalmente, el modelo parte de la suposición de que no existe línea de vista entre el transmisor y el receptor, de manera que la señal recibida se debe a las señales que han seguido múltiples trayectos [4].

La potencia promedio de los multi-trayectos es estacionaria sólo sobre secciones cortas de la ruta del dispositivo móvil y sobre secciones más largas varia lentamente debido al desvanecimiento. Este comportamiento se puede representar por la combinación de una distribución Rayleigh con una distribución Log-normal. Esta distribución mezclada es comúnmente denominada distribución Suzuki.

Localmente las variaciones en la amplitud de la señal son Rayleigh distribuidas con parámetro $\sigma$ (ecuación (1)) [5].

$$
f(r \mid \sigma)=\frac{r}{\sigma^{2}} \exp \left(\frac{-r^{2}}{2 \sigma^{2}}\right)(r \geq 0)
$$

Esto es válido solo sobre secciones cortas de la ruta del móvil, es decir, alrededor de unas diez a cien longitudes de onda. La expresión $f(r \mid \sigma)$ se refiere a la distribución condicional de $r$ con 
respecto a $r$. El parámetro $\sigma$ sigue una distribución log-normal sobre secciones más largas de la ruta del móvil.

Dada una variable aleatoria, $\mathrm{X}$, que sigue una distribución gaussiana cuya función de distribución de probabilidad está dada por la ecuación (2).

$$
p(X)=\frac{1}{\sigma \sqrt{2 \pi}} \exp \left[-\frac{1}{2}\left(\frac{X-m}{\sigma}\right)^{2}\right]
$$

Donde $\mathrm{m}$ es la media y $\sigma$ la desviación estándar, si la variable aleatoria $x$ es tal que $X=\ln (x)$, entonces $x$ sigue una distribución log-normal con función de distribución de probabilidad dada por la ecuación (3).

$$
p(X)=\frac{1}{\sigma \sqrt{2 \pi}} \frac{1}{x} \exp \left[-\frac{1}{2}\left(\frac{\ln (x)-m}{\sigma}\right)^{2}\right]
$$

Esta distribución permite describir potencias, voltajes o fuerzas de campo en unidades lineales. Sin embargo, es de interés el voltaje o potencia en dB. Así, cuando la variable es el voltaje, su función de distribución de probabilidad dada por la ecuación (4).

$$
f(v)=\frac{8,686}{v \sqrt{2 \pi} \Sigma} \exp \left\{-\frac{[20 \log (v)-M]^{2}}{2 \Sigma^{2}}\right\}
$$

Donde ahora la media, M, y la desviación estándar, $\Sigma$, están en dB.

Volviendo a la distribución Suzuki [6], el parámetro $\sigma$ se asume constante sobre áreas pequeñas y varía de acuerdo a una distribución log-normal sobre áreas más grandes (ecuación (5)).

$$
f(\sigma)=\frac{8,686}{\sigma \sqrt{2 \pi} \Sigma} \exp \left\{-\frac{[20 \log (\sigma)-M]^{2}}{2 \Sigma^{2}}\right\}
$$

Se escribe la expresión completa y se elimina la variable $\sigma$ a través de la integración (ecuación (6) y ecuación (7)).

$$
f(r)=\int_{0}^{\infty} f(r \mid \sigma) f(\sigma) d \sigma
$$

$f(\mathrm{r})=\frac{8,686}{\sum \sqrt{2 \pi}} \int_{0}^{\infty} \frac{1}{\sigma^{3}} \exp \left[-\frac{[20 \log (\sigma)-M]^{2}}{2 \Sigma^{2}}\right]$

\section{TRABAJOS REALIZADOS}

Algunos trabajos han realizado modificaciones al modelo Suzuki original buscando modelar con mayor precisión los fenómenos que se presentan en el entorno real. Así por ejemplo, en [7] se propone modificar el modelo introduciendo correlación cruzada entre los procesos normales de la parte de Rayleigh debido a que a menudo la suposición de independencia estadística no cumple con las condiciones reales de propagación cuando se presenta el fenómeno de multi-trayecto.

Es evidente que el proceso de Suzuki es un modelo estadístico adecuado para describir un canal móvil terrestre, donde a menudo se asume que una línea directa de visión entre componentes está ausente. Pero para los canales terrestres móviles por satélite, donde la mayor parte del tiempo una línea directa de visión entre componente está presente, una extensión del proceso de Suzuki es indispensable. Por lo tanto, en [8] se ha propuesto un modelo de distribución de probabilidad que es una combinación de un proceso Rice y un proceso Lognormal. En este modelo estadístico, el proceso log-normal modela las variaciones de la señal a largo plazo debido al efecto de sombreado que afecta tanto a la línea directa de visión de los componentes y los componentes dispersos.

De manera similar, en [9] se propone un modelo Suzuki extendido. El proceso Suzuki extendido también está definido por el producto de un proceso Rice y un proceso log-normal, pero la fase y cuadratura de los componentes que describen el proceso Rice se les permite ser mutuamente correlacionada, lo cual es equivalente a considerar densidades de potencia Doppler espectral asimétricas. Por otra parte, se incluyen en el modelo la situación más general donde la línea directa de visión es desplazada en frecuencia debido al efecto Doppler. 
En [10] el modelo de Suzuki es generalizado con un proceso Nakagami en lugar de utilizar la distribución Rayleigh. La distribución Nakagami es de particular interés porque incluye Rayleigh como un caso especial y también porque puede ser utilizado como aproximación a la distribución Rice.

Por otra parte, debido a la complejidad de la integral que presenta el modelo Suzuki, se han propuesto métodos alternativos que permiten realizar simulaciones con menor complejidad y costos computacionales bajos [11] Una de estas formas alternativas consiste en la generación de secuencias aleatorias Suzuki distribuidas [12] evitando así la realización de costosas pruebas hardware en sistemas de comunicaciones inalámbricas.

\section{PREDICCIÓN DE PROPAGACIÓN}

\subsection{Mediciones y caracterización del Entorno}

Con el fin de caracterizar el entorno y validar el modelo, se realizaron mediciones durante 10 horas usando la configuración de la figura 1 bajo las condiciones mostradas en la tabla 1.

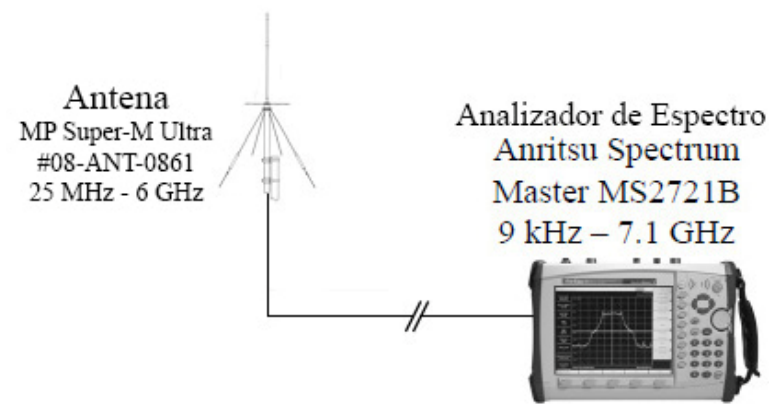

Figura 1. Diagrama de medición

Los datos obtenidos se muestran en la figura 2 en donde se observa el nivel de la señal recibida en función del tiempo y la frecuencia.

Con los datos medidos, se calculó la media M y la desviación estándar $\Sigma$ para cada una de las frecuencias muestreadas. Posteriormente como se explicará en la sección IV-B, cada me- dia y desviación estándar se toman como parámetros de entrada para generar la respectiva serie de tiempo que permitirá realizar una simulación y predicción de propagación.

Tabla 1. Parámetros de medición

\begin{tabular}{|c|c|}
\hline $\begin{array}{c}\text { RBW } \\
\text { (Anchodebanda de resolución) }\end{array}$ & $3 \mathrm{kHz}$ \\
\hline $\begin{array}{c}\text { VBW } \\
\text { (Ancho de banda de video) }\end{array}$ & $1 \mathrm{kHz}$ \\
\hline fmín & $850 \mathrm{MHz}$ \\
\hline fmáx & $900 \mathrm{MHz}$ \\
\hline Latitud & $4^{\circ} 37^{\prime} 41^{\prime \prime} \mathrm{N}$ \\
\hline Longitud & $74^{\circ} 3^{\prime} 57^{\prime \prime} \mathrm{W}$ \\
\hline Temperatura & $12^{\circ} \mathrm{C}$ \\
\hline Altura & $2641 \mathrm{~m}$ \\
\hline Pisoderuido & $-110 \mathrm{dBm}$ \\
\hline
\end{tabular}

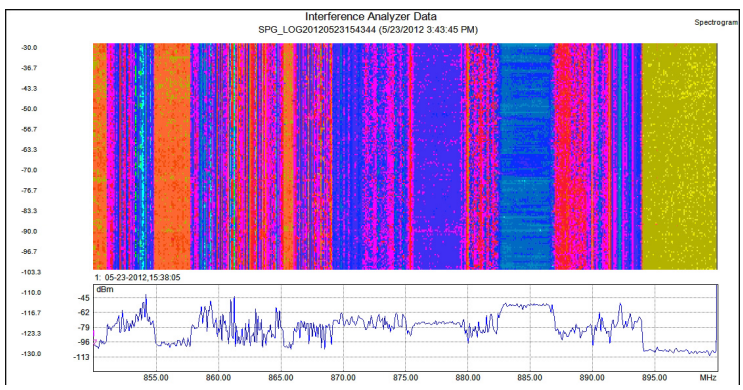

Figura 2a. Datos medidos: tiempo vs frecuencia

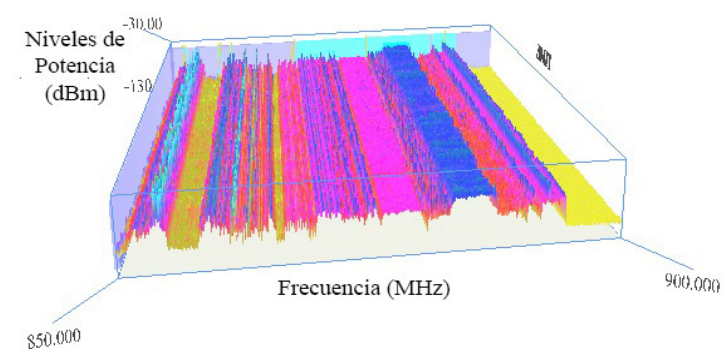

Figura 2b. Datos medidos: Potencia vs tiempo vs frecuencia

\subsection{Simulación del entorno}

Se desea generar una serie de tiempo Suzuki distribuida. Usando MATLAB® se genera una serie de tiempo de acuerdo al diagrama en la figura 3. 


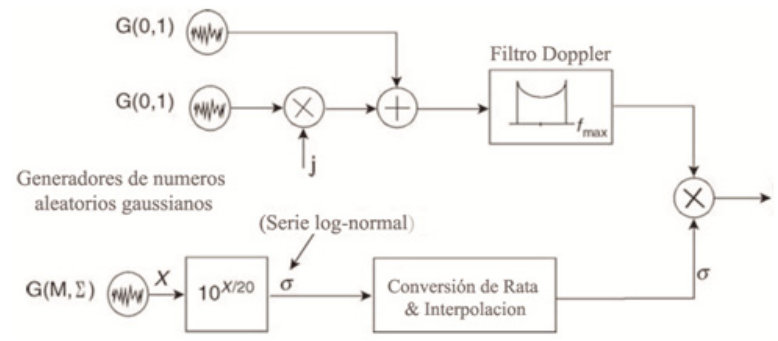

Figura 3. Diagrama del generador de la serie de tiempo

La parte superior en la figura 3 representa una serie de tiempo Rayleigh distribuida: dos series gaussianas con media cero y desviación estándar unitaria en cuadratura, que posteriormente atraviesan un filtro que simula el efecto Doppler en la señal y limita el ancho de banda del proceso de desvanecimiento.

La parte inferior en la figura 3 representa una serie de tiempo log-normalmente distribuida: donde $X=\ln (\sigma)$, sigue un distribución gaussiana con media $M$ y desviación estándar $\Sigma$. Como se mencionó antes, cada media My desviación estándar $\Sigma$ fueron encontradas previamente a partir de los datos medidos (los primeros $5 \mathrm{mi}$ nutos) para cada una de las frecuencias muestreadas.

Las series de tiempo de los dos caminos, muestreados a la misma tasa, son multiplicadas para producir una serie de tiempo Suzuki distribui$\mathrm{da}$, que mantiene las tasas de cambio tanto del efecto de sombreado (interferencias) como las del fenómeno de multi-trayectoria. Es decir, las variaciones lentas y rápidas respectivamente.

Una serie de tiempo Suzuki distribuida es generada para cada una de las frecuencias muestreadas durante las mediciones del entorno real mostradas en la sección cuatro. Los resultados de simulación obtenidos para la predicción de las primeras 5 horas se muestran en la figura 4 .

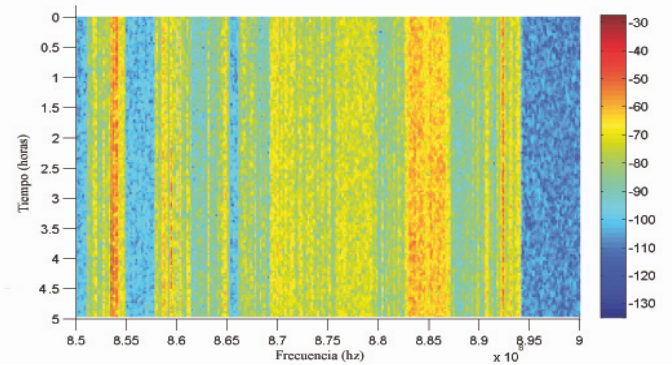

(a)

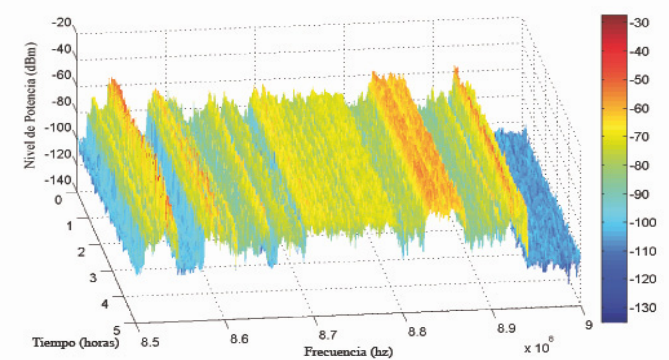

(b)

Figura 4. Datos simulados

(a) Tiempo vs frecuencia

(b) Potencia vs tiempo vs frecuencia

\section{RESULTADOS}

Para validar el modelo, se realizó una comparación estadística calculando el error cuadrático medio entre los datos medidos y los datos obtenidos con el modelo. La figura 5 muestra el error encontrado en cada una de las frecuencias muestreadas para el primer tramo de predicción. La tabla 2 resume los errores en promedio para cada tramo de predicción. Dichos errores varían alrededor de los $5 \mathrm{dBm}$ que corresponden en promedio al $6 \%$ con respecto a la media de la señal recibida. Por lo tanto, dado que se ha demostrado que el modelo arroja valores muy cercanos a los obtenidos en el entorno real, se comprueba la validez del modelo. 


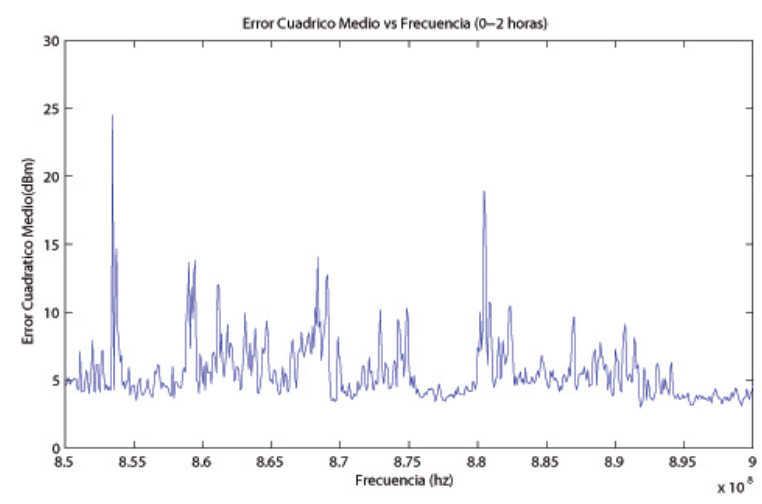

Figura 5. Error cuadrático medio vs frecuencia (0-2 horas)

Tabla 2. Error cuadrático medio por tramo de repetición

\begin{tabular}{|c|c|}
\hline Tiempo (horas) & Error cuadrático medio $(\mathrm{dBm})$ \\
\hline $0-2$ & 4.91 \\
\hline $2-4$ & 5.01 \\
\hline $4-6$ & 4.87 \\
\hline $6-8$ & 4.76 \\
\hline $8-10$ & 4.82 \\
\hline
\end{tabular}

Referencias Bibliográficas

[1] K. Tapan, K. Zhong, J. Kyungjung, M. Abdellatif, M. S. Asurvey of various propagation models for mobile communication, IEEE Antennas and Propagation Magazine, Vol. 45, No. 3, pp 51-82, June 2003.

[2] L. Pedraza, C. Hernández, D. Ballesteros. Análisis experimental para un modelo de propagación de la telefonía móvil de la Universidad Militar, Ciencia e Ingeniería Neogranadina, Vol. 19, No. 2, pp 45-58, 2009.

[3] L. Pedraza, D. Ballesteros, H. Vacca. Estudio de modelos de propagación en el entorno de la Universidad Distrital Francisco José de Caldas, Visión Electrónica: Algo Más Que Un Estado Sólido, Vol. 4, No. 2, pp 77-87, 2010.

[4] F. Perez, P. Marino. Modeling the wireless propagation channel. Editorial John Wiley

\section{CONCLUSIONES}

Se ha presentado la simulación de medidas de propagación de un canal inalámbrico a través del modelo Suzuki. Los datos obtenidos bajo simulación fueron comparados estadísticamente con las mediciones en el entorno real. Los resultados obtenidos muestran un pequeño error que se atribuye al comportamiento del usuario final que varía dependiendo la hora y el día de la semana. No obstante, como se observa en la tabla 2, la magnitud del error es lo suficientemente menor como para demostrar la validez del modelo. Así, se demuestra que es posible hacer uso del modelo Suzuki como una herramienta para el diseño de redes móviles o predicción de usuarios primarios y análisis de la ocupación del canal en redes de radio cognitivo. En futuros trabajos se podría minimizar el error sintonizando automáticamente los parámetros de entrada al modelo: media M y desviación estándar $\Sigma$, a través de algoritmos de inteligencia computacional que permitirían ajustar los parámetros teniendo en cuenta las variaciones en el comportamiento del usuario móvil.

[5] F. Andreas, Wireless comumnications. Editorial John Wiley \& Sons Ltd, 2011.

[6] H. Suzuki, A statistical model for urban radio propagation. IEEE Transactions on Communicatios, Vol. 25, No. 7, pp 213225, 1979.

[7] A. Krantzik, D. Wolf, Analysis of a modified Suzuki fading channel model, in Acoustics, Speech, and Signal Processing. ICASSP-89, Vol.4, pp 2250 -2253, 1989.

[8] G. Corazza, F. Vatalaro, A statistical model for land mobile satellite channels and its application to nongeostationary orbit systems. Vehicular Technology, IEEE Transactions on, Vol. 43, No. 3, pp $738-742,1994$.

[9] M. Patzold, U. Killat, F. Laue. An extended Suzuki model for land mobile satellite channels and its statistical 
properties, Vehicular Technology, IEEE Transactions on, Vol. 47, No. 2, pp 617630, 1998.

[10] F. Ramos A, V. Kontorovitch, M. Lara. Generalization of Suzuki model for analog communication channels, in Antennas and Propagation for Wireless Communications, 2000 IEEE-APS Conference on, pp $107-110,2000$.

[11] M. Patzold, U. Killat, F. Laue. A deterministic digital simulation model for Suzuki processes with application to a shadowed Rayleigh land mobile radio channel, Vehicular Technology, IEEE Transactions on, Vol. 45, No. 2, pp 318 $-331,1996$.

[12] A. Jakubiak. Modelling and simulation of urban multipath propagation, in Microwaves, Radar and Wireless Communications. 